\title{
Shifting the paradigm of peri-oprative care in colorectal surgery in a Sri Lankan tertiary care unit
}

\author{
Bawantha Gamage \\ Department of Surgery, University of Sri Jayewardenepura, Sri Lanka
}

Keywords: Colorectal surgery; enhanced recovery after surgery; peri-operative care; pelvic MRI

\begin{abstract}
Enhanced recovery after colorectal surgery (ERAS) protocol is a multimodal perioperative care pathway aiming to reduce stress response to surgery. It helps to return to normal physiology at earliest with minimum complications compared to traditional care pathway in colorectal surgery. In order to have intended benefits of ERAS, accurate staging of the patients with colorectal cancer is invaluable. Pretreatment pelvic MRI scan is the gold standard in rectal cancer staging due to its proven benefits.
\end{abstract}

While preoperative MRI scan in rectal cancer patients with internationally recognized reporting protocol has been encouraged, ERAS with some modifications (mERAS) has been introduced at the university surgical unit of the Colombo south teaching hospital to overcome the resource limitations at local setting.

Modified ERAS protocol was well tolerated by our cohort of patients and the favourable outcomes of ERAS protocol in our centre are comparable with published data. Therefore mERAS would be a safe alternative to the traditional practice of colorectal surgery in Sri Lanka.

This article describes the methods of mERAS implementation and the strategies adhered in shifting the paradigm of perioperative care in colorectal surgery at our centre.

\section{Introduction}

The outcome of colorectal surgery for cancer depends on the operative technique, accurate local staging and evidence based peri operative management. In traditional practice of rectal cancer staging in Sri Lanka, contrast enhanced computerised tomographic scan (CECT) of the pelvis has been the investigation used in local staging of rectal cancers in most of the centres; magnetic resonance imaging (MRI)has not been widely employed mainly due to non-availability. In

Correspondence: Bawantha Gamage
E-mail: bawantha@sjp.ac.lk
Received: 15-11-2018 Accepted: 27-12-2018
(iD) http://orcid.org/0000-0003-4381-228X CC C
DOI: http://doi.org/10.4038/sljs.v36i4.8552 C)

The Sri Lanka Journal of Surgery 2018; 36(4): 6-10 addition preoperative prolonged fasting, use of oral mechanical bowel preparation, nasogastric tubes, routine drains and prolonged postoperative 'nil by mouth' regimes have been considered to be essential. The average hospital stay after traditional practice of colorectal surgery has historically been reported to be between 7 and 12 days [1,2].

The recommendation for local staging of patients with rectal cancer is MRI of the pelvis [3]. The MERCURY study group in rectal cancer has shown that high resolution MRI will accurately predict the involvement of the surgical resection margin in rectal cancer [4]. The result of this investigation will determine whether the patient would need neoadjuvant treatment prior to surgery. Making management decisions without accurate staging would lead to delay in recovery and poor outcome. Therefore, steps taken to enhance postoperative recovery should be preceded by accurate staging of the disease.

The concept of augmented postoperative recovery was first described by Danish Professor Henrik Kehlet in the late 1990s although he did not use the term "Enhanced Recovery After Surgery" [5].He extensively studied on pathophysiology of surgery, attenuating surgical stress, multimodal analgesia in managing postoperative pain and employed a protocol in managing patients in the peri-operative period $[5,6]$. The work led to the concept of "Fast-Track-Surgery".

The principal aim of the ERAS protocol is to attenuate the stress response to surgery by optimizing the patient's normal physiology. This resulted in speeding up recovery and promoted early return to normal activities [6]. Several alterations to the original protocols have been added with the introduction of minimally invasive surgery and accumulating new evidence. A group of academic surgeons who worked tirelessly on this subject formed the ERAS society in early 2000. Their mission was to "develop perioperative care and to improve recovery through research, education, audit and implementation of evidence-based practice"[7].

ERAS has been associated with reduced postoperative hospital stay to 3-5 days [5] and early return to normal activity with low complication rates [8] without compromising oncological outcome. Summary of steps of ERAS is outlined in Table $1[9,10]$. 


\begin{tabular}{|rl|}
\hline \multicolumn{2}{|l|}{ Table l- Summary of ERAS } \\
\hline Preoperative interventions \\
\hline 1. & Preoperative patient education and counselling \\
\hline 2. & Optimizing comorbidities \\
\hline 3. & Selective use of bowel preparation/ use of antibiotic \\
& associated mechanical bowel preparation \\
\hline 4. & Minimal preoperative fasting \\
\hline 5. & Preoperative thromboembolism prophylaxis \\
\hline 6. & Preoperative carbohydrate loading \\
\hline Perioperative interventions \\
\hline 1. & Minimally invasive surgery \\
\hline 2. & Antibiotic prophylaxis \\
\hline 3. & Avoid opioids \\
\hline 4. & Postoperative nausea and vomiting prophylaxis \\
\hline 5. & Prevent hypothermia \\
\hline 6. & Goal directed fluid therapy \\
\hline 7. & Avoid routine drains and nasogastric tubes \\
\hline Postoperative interventions \\
\hline 1. & Early and progressive mobilization \\
\hline 2. & Early oral feeding \\
\hline 3. & Early removal of drains/ catheters/ NG tubes \\
\hline & Early discharge \\
\hline
\end{tabular}

Though evidence based, ERAS has yet to be firmly established in Sri Lanka. One reason may be the limitations of resources to follow all steps of the protocol. The ERAS protocol was initiated with some modifications at the University Surgical Unit of the Colombo South Teaching Hospital with the aim of assessing the outcome of mERAS among our patient cohort.

\section{Methodology \\ Preoperative staging pelvic MRI in rectal cancer patients} To stage rectal cancer accurately, an MRI of the pelvis was performed and was reported according to standard protocol, which highlighted the circumferential resection margin (CRM), extramural vascular invasion (EMVI) and the lateral pelvic side wall involvement (LPSWI) [11].

Management decisions were made at the colorectal multidisciplinary team (MDT)meetings after discussing the result of pelvic MRI and CECT of the abdomen, amongst other criteria such as fitness for anaesthesia. This was considered avital step in management in order to subject the patients to the modified ERAS protocol.

\section{Application of modified ERAS (mERAS) protocol :}

All steps of ERAS were not possible in Sri Lanka due to resource limitations. While preserving the principles of ERAS, the following modifications were made to overcome the limitations in our local setting (table 2).

All other steps of the standard ERAS have been followed accordingly.

The introduction of this prospective descriptive study of mERAS at the University Surgical Unit, CSTH, which was designed to evaluate the outcome of patients having surgery for colorectal cancer, was approved by the Ethics Review Committee of the Faculty of Medical Sciences University of Sri Jayewardenepura.

\section{Patient recruitment}

All patients were admitted under the care of a single surgeon for elective colorectal resections, and who gave informed consent to follow the mERAS protocol, were included in the study. Patients who required emergency colorectal resections were excluded.

\begin{tabular}{|l|l|}
\hline \multicolumn{2}{|l|}{ Table 2. Modified steps of ERAS protocol } \\
\hline Standard ERAS protocol & Modifications we made at CSTH \\
\hline Admit on the day of the surgery & Admit on the day prior to surgery \\
\hline $\begin{array}{l}\text { Epidural for open but not for laparoscopic resections, free } \\
\text { use of patient controlled anaesthesia (PCA) }\end{array}$ & $\begin{array}{l}\text { Frequent use of epidural anaesthesia (due to lack of patient-controlled } \\
\text { analgesia devices) }\end{array}$ \\
\hline Intra operative non-invasive goal directed fluid therapy & Intraoperative CVP guided fluid therapy \\
\hline NG tubes are not recommended & NG tube was inserted and kept for 24 hours \\
\hline No drains recommended & $\begin{array}{l}\text { Selective use of drains in low anterior resection (AR), proctocolectomy } \\
\text { and abdominoperineal resection (APR). }\end{array}$ \\
\hline Post op Routine ICU care not recommended & Routine post op ICU/HDU care given in the first 24 hours postoperatively. \\
\hline $\begin{array}{l}\text { Early discharge from hospital } \\
\text { (3-5days) }\end{array}$ & $\begin{array}{l}\text { Discharge after normal diet is consumed and when mobile } \\
5-6 \text { days, due to the paucity of community based care. }\end{array}$ \\
\hline
\end{tabular}

The Sri Lanka Journal of Surgery 2018; 36(4): 6-10 


\section{Data collection and analysis}

Data on preoperative preparation, surgery, postoperative care and complications have been recorded in a colorectal data base in all the patients included in this study. Data from 2011 to 2017 were evaluated. Analysis was done using SPSS data software( IBM SPSS Statistics for Windows, Version 23.0. Armonk, New York, USA: IBM Corp.)

\section{Results}

\section{Demographic data}

One hundred and twelve patients (median age - 60 years, range 23 to 88 years) underwent colon and rectal cancer resection during this study period. The ratio of male to female was 1.08: 1.

\section{Mode of surgery}

Laparoscopic resections were performed in $71.4 \%$ of the study cohort (Figure. 1). The overall conversion rate was 8.9 $\%$. The number of laparoscopic resections performed per year has increased over time. (Figure.2).

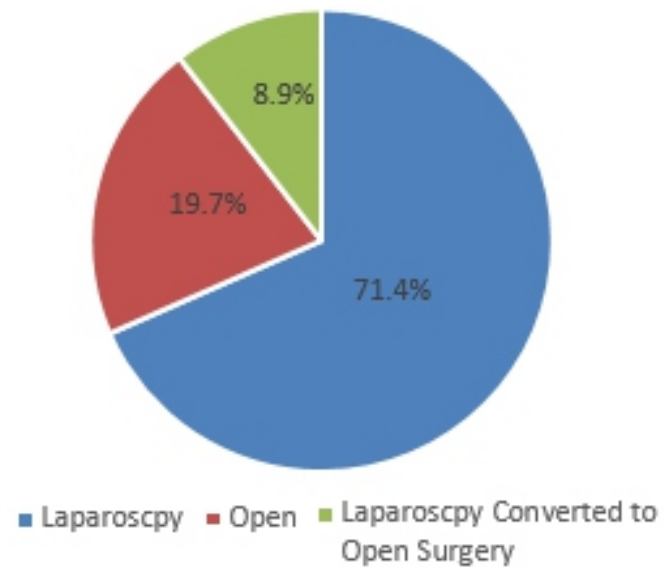

Figure 1. Method of resection

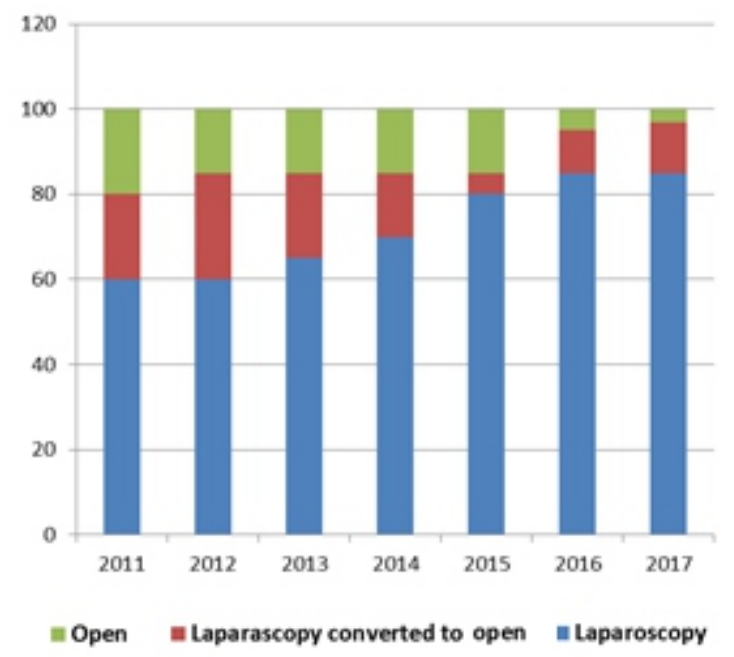

Figure 2. Trend of modes of surgery

\section{Staging pelvic MRI scan in patients with rectal cancer}

MRI was performed in rectal cancer patients for accurate local staging of the disease. The percentages have been increasing over the years, and now, more than $90 \%$ of patients with rectal cancer will have a pelvic MRI as the investigation of choice in local staging provided they do not have contraindications (Figure 3).

\section{Adherence to the steps of ERAS}

All patients were admitted one day prior to surgery as lack of proper transport facility did not allow same day admission. All the patients were educated on the perioperative care plan and their concerns were acknowledged. Preoperative thromboembolism prophylaxis was started with single dose of subcutaneous low molecular weight heparin in all patients.

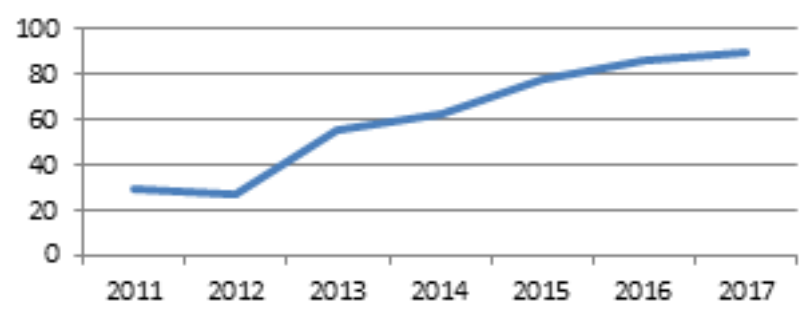

Figure 3. Percentage of MRI Scans performed for rectal cancers

A phosphate enema was administered early on the morning of the day of surgery in the case of left colonic and rectal resections $(84 \%)$. No bowel preparation was used in right and transverse colon resections. Patients were requested to fast for a period of 6 hours for solids and 2 hours before surgery for clear fluids. Carbohydrate loading was given on the previous day of surgery as well as 2 hours prior to surgery. Antibiotic prophylaxis was given to all the patients prior to the induction of anaesthesia. Epidural anaesthesia was used frequently due to the unavailability of patient controlled anaesthesia devices. It was used in all open resections(19.7\%). CVP guided fluid therapy was used instead of goal directed fluid therapy as oesophageal dopp-ler monitoring facilities were not available. Intraperitoneal drains were inserted in all cases of low anterior resection (48\%), abdominoperineal resection and total proctocolec-tomy. The median number of days to resume normal diet, mobilise, removal of the urinary catheter and drains, including stay in intensive care are shown in Figure. 4.

Oral fluids were started by the evening of the same day of surgery, and normal diet the following day, in the absence of post-operative nausea and vomiting. By day 3 after surgery, $70 \%$ of patients had a bowel movement. The median duration of hospital stay was 5 days (range 3-21 days).

The Sri Lanka Journal of Surgery 2018; 36(4): 6-10 


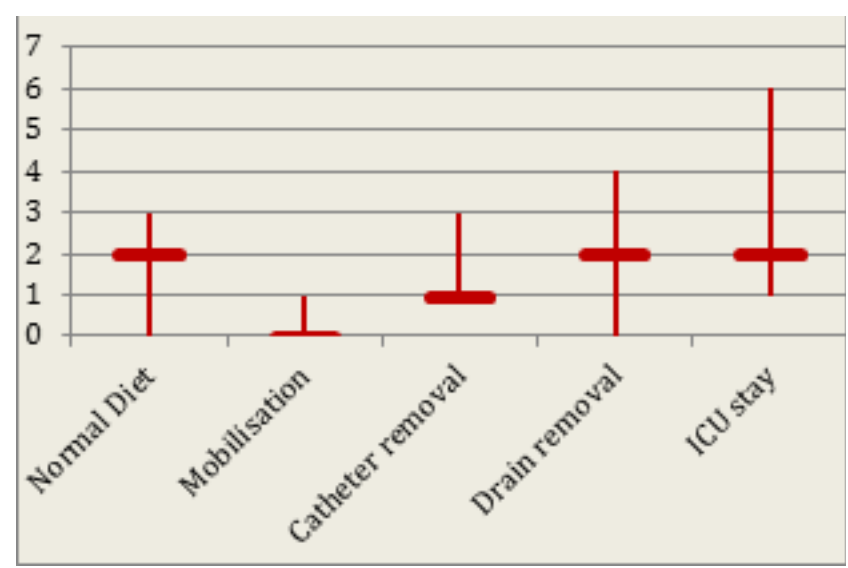

Figure 4. Post-operative care (Median days)

\section{Postoperative complications}

In the first 30 days after surgery, the following complications (Clavien-Dindo $>2$ ) were reported in our cohort; surgical site infection $-17.85 \%$, respiratory tract infection- $4.4 \%$, urinary tract infection $-2.6 \%$ and anastomotic leaks $-3.5 \%$. All anastomotic leaks were in patients who underwent low AR $(n=4)$, and who had had neoadjuvant chemo-radiotherapy. All leaks were identified within the first 5 days post-operation; three required re-laparotomy. 30-Day mortality was zero in this cohort.

\section{Discussion}

\section{Role of preoperative MRI scan in rectal cancer}

The MERCURY study group demonstrated the accuracy of pelvic MRI in delineating the anatomy of mesorectal fascia and future surgical clearance [4]. Also, the study showed that preoperative prognostication can be done by accurate measurement of extramural tumor spread [12, 13]. The National Comprehensive Cancer network (NCCN) guideline on rectal cancer recommends preoperative pelvic MRI scans in all patients with rectal cancer [3]. To be an effective tool in preoperative evaluation, standardization of the technique of MRI and reporting is vital [11]. In our study the number of patients having pre-operative pelvic MRI for rectal cancer has been increasing, which has helped the predictability at our MDT meetings.

\section{Outcome of mERAS at local setting}

Modified ERAS protocol has been well tolerated by our cohort of patients. Published data on this aspect in local setting is scarce and we could not find similar studies to compare with our results. Similar studies with ERAS protocol have been published from developed countries. One metaanalysis, which included 6 randomized control trials comparing conventional practice and ERAS in open colorectal surgery, has mentioned that only a mean of 9 (range- 4 to 12) elements of the ERAS protocol were used in those trials [8]. This meta-analysis has shown that ERAS is associated with reduced time of hospital stay and postoperative complication rates after open colorectal surgery without compromising patient safety [8]. In our study group, the modifications were done while sticking to the principles of ERAS, in order to overcome the limitation of resources at local setting.

A Japanese study of 257 patients undergoing ERAS protocol, has shown reduction of postoperative hospital stay by 3 days from 10 days to 7 days. This was a statistically significant difference, without adversely affecting the short term outcome [14]. Reduction of postoperative hospital stay to a median of 4 days has been reported with the implementation of ERAS in a study from Belgium [15]. All procedures were laparoscopic with a $9.5 \%$ conversion rate and a reported 2.1 $\%$ anastomotic leaks. The authors have suggested widespread implementation of ERAS principles for cost effective use of healthcare [15]. A larger Italian study comprising 1065 patients reported an average 3 days of hospital stay with no difference in complication rates [16].

Our results of implementing ERAS with modifications are comparable with these published data. The modifications that we undertook helped overcome resource limitations at our local setting.

\section{How this paradigm shift was made possible}

Initiation of a change is always a challenge, and sustenance of change is even more challenging. The following approaches were adopted to overcome these challenges and to make the protocol sustainable.

1. Formal introduction on modified ERAS was given to both the ward staff and the anaesthetic team. All steps were properly explained. The advantages of the new protocol were highlighted.

2. Laparoscopy facilities at our centre were upgraded.

3. Adedicated stoma nurse was trained. The nurse was able to build good rapport with the patient and trained the patient as well as the patient's immediate care takers.

4. The unit protocol was clearly drafted and distributed among the staff. All their concerns were acknowledged and implemented if appropriate.

5. Patient information leaflets and consent forms were distributed to the patients prior to the surgery.

6. A system was developed to record data of all procedures which enabled frequent evaluation of the unit protocol.

7. A Colorectal Multi-Disciplinary Team (MDT) meeting was started, which involved surgeons, pathologists, radiologists, an oncologist and a stoma care nurse.

8. Two books were published with the aim of educating patients and the public on colorectal diseases and the ERAS programme. 


\section{Conclusions}

We recommend staging pelvic MRI as the investigation of choice for local staging in rectal cancer, which should be reported by radiologists trained in rectal cancer MRI, and which should be the focal point of discussion at a colorectal MDT. The modified ERAS protocol was well tolerated by our cohort of patients and the favourable outcomes of ERAS at our centre were comparable with published data. The adoption of the ERAS protocol with some modifications to suit the local setting would be a development to current traditional practice of colorectal surgery for colorectal cancer in Sri Lanka.

All authors disclose no conflict of interest. The study was conducted in accordance with the ethical standards of the relevant institutional or national ethics committee and the Helsinki Declaration of 1975, as revised in 2000 .

\section{References}

1. Schoetz DJ, Bockler M, Rosenblatt MS, Malhotra S, Roberts PL, Murray JJ, Coller JA, Rusin LC. "Ideal" length of stay after colectomy. Diseases of the colon \& rectum. $1997 \mathrm{Jul}$ 1;40(7):806-10. https://doi.org/10.1007/bf02055437

2. Retchin SM, Penberthy L, Desch C, Brown R, Jerome-D'Emilia $\mathrm{B}$, Clement D. Perioperative management of colon cancer under Medicare risk programs. Archives of internal medicine. 1997 Sep 8;157(16):1878-84.

https://doi.org/10.1001/archinte.1997.00440370126013

3. Engstrom PF, Arnoletti JP, Benson AB, Chen YJ, Choti MA, Cooper HS, Covey A, Dilawari RA, Early DS, Enzinger PC, Fakih MG. Rectal cancer. Journal of the National Comprehensive Cancer Network. 2009 Sep 1;7(8):838-81. https://doi.org/10.6004/jncen.2009.0057

4. MERCURY Study Group. Diagnostic accuracy of preoperative magnetic resonance imaging in predicting curative resection of rectal cancer: prospective observational study. Bmj. 2006 Oct 12;333(7572):779. https://doi.org/BMJ 2006;333:779

5. Kehlet H. Multimodal approach to control postoperative pathophysiology and rehabilitation. British journal of anaesthesia. 1997 May 1;78(5):606-17.

https://doi.org/10.1093/bja/78.5.606

6. Basse L, Jakobsen DH, Billesbølle P, Werner M, Kehlet H. A clinical pathway to accelerate recovery after colonic resection. Annals of surgery. $2000 \mathrm{Jul} ; 232(1): 51$. http://erassociety.org/about/history

8. Varadhan KK, Neal KR, Dejong CH, Fearon KC, Ljungqvist O, Lobo DN. The enhanced recovery after surgery (ERAS) pathway for patients undergoing major elective open colorectal surgery: a meta-analysis of randomized controlled trials. Clinical nutrition.
2010 Aug 1;29(4):434-40.

https://doi.org/10.1016/j.clnu.2010.01.004

9. Carmichael JC, Keller DS, Baldini G, Bordeianou L, Weiss E, Lee L, Boutros M, McClane J, Feldman LS, Steele SR. Clinical practice guidelines for enhanced recovery after colon and rectal surgery from the American Society of Colon and Rectal Surgeons and Society of American Gastrointestinal and Endoscopic Surgeons. Diseases of the Colon \& Rectum. 2017 Aug 1;60(8):761-84 https://doi.org/10.1097/DCR.0000000000000883

10. Lassen K, Soop M, Nygren J, Cox PB, Hendry PO, Spies C, von Meyenfeldt MF, Fearon KC, Revhaug A, Norderval S, Ljungqvist $\mathrm{O}$. Consensus review of optimal perioperative care in colorectal surgery: Enhanced Recovery After Surgery (ERAS) Group recommendations. Archives of surgery. 2009 Oct 19;144(10):961-9.

https://doi.org/10.1001/archsurg.2009.170

11. Taylor F G, Swift R I, Blomqvist L, Brown G. A systematic approach to the interpretation of preoperative staging MRI for rectal cancer. American Journal of Roentgenology. 2008 Dec;191(6):1827-35. https://doi.org/10.2214/AJR.08.1004

12. MERCURY Study Group. Extramural depth of tumor invasion at thin-section MR in patients with rectal cancer: results of the MERCURY study. Radiology. 2007 Apr;243(1):132. https://doi.org/10.1148/radiol.2431051825

13. Taylor FG, Quirke P, Heald RJ, Moran B, Blomqvist L, Swift I, Sebag-Montefiore DJ, Tekkis P, Brown G. Preoperative highresolution magnetic resonance imaging can identify good prognosis stage I, II, and III rectal cancer best managed by surgery alone: a prospective, multicenter, European study. Annals of surgery. 2011 Apr 1;253(4):711-9.

https://doi.org/10.1097/SLA.0b013e31820b8d52

14. Shida D, Tagawa K, Inada K, Nasu K, Seyama Y, Maeshiro T, Miyamoto S, Inoue S, Umekita N. Enhanced recovery after surgery (ERAS) protocols for colorectal cancer in Japan. BMC surgery. $2015 \mathrm{Dec} ; 15(1): 90$. https://doi.org/10.1186/s12893-015-0079-0

15. Fierens J, Wolthuis AM, Penninckx F, D'Hoore A. Enhanced recovery after surgery (ERAS) protocol: Prospective study of outcome in colorectal surgery. Acta Chir Belg. 2012;112(5):355-8.

https://doi.org/10.1080/00015458.2012.11680851

16. Lucchi A, Berti P, Gabbianelli C, Siani L, Vitolo GC, Grassia M, Vandi F, Maffi MF, Garulli G. Eras in laparoscopic colorectal surgery: 8 years of experience. European Journal of Surgical Oncology. 2016 Oct 1;42(10):S216. https://doi.org/10.1016/j.ejso.2016.04.050 